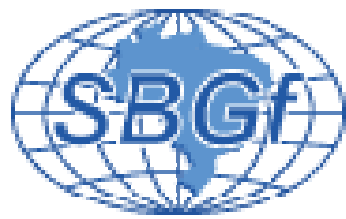

\title{
Diferentes parametrizações de modelos de falha geológica e bacia sedimentar na modelagem acústica 2-D
}

Roberto Hugo M. dos Santos e Wilson M. Figueiró. CPGG - UFBA, LAGEP, Salvador, Bahia, Brasil.

Copyright 2006, SBGf - Sociedade Brasileira de Geofísica

Este texto foi preparado para a apresentação no II Simpósio de Geofísica da Sociedade Brasileira de Geofísica, Natal, 21-23 de setembro de 2006. Seu conteúdo foi revisado pela Comissão Tecno-científica do II SR-SBGf mas não necessariamente representa a opinião da SBGf ou de seus associados. E proibida a reprodução total ou parcial deste material para propósitos comerciais sem prévia autorização da SBGf.

\section{Resumo}

O algoritmo desenvolvido neste trabalho é baseado no método das diferenças finitas aplicado à equação acústica da onda. A modelagem sísmica numérica foi implementada empregando malhas regulares. Os modelos foram apresentados por dois tipos distintos de parametrizações: por blocos e polinomial trigonométrica. São apresentadas simulações da propagação de ondas em dois modelos distintos, representados pelas duas parametrizações mencionadas, possibilitando, assim, a geração de sismogramas sintéticos para comparação. Os sismogramas obtidos, com a parametrização polinomial, mostram alguns comportamentos não desejados, por outro lado, como vantagens, temos: economia de espaço de memória, produção de modelos de velocidades suavizados em tempo, entre outras.

\section{Introdução}

A modelagem sísmica numérica: é vista como a resolução do problema direto em sua formulação sísmica, participa dos procedimentos de inversão sísmica que requer uma teoria para a simulação da propagação de ondas sísmicas gerando dados sintéticos, é útil nos processos de migração, pode ser utilizada na interpretação sísmica de regiões com geologia complexa, e gera dados para teste de algoritmos de processamento. Tradicionalmente, utiliza-se simplificações das equações da onda, tais como: aproximações assintóticas e linearizações. A teoria do raio (Červený, Molotov e Pšenčík, 1977; Keller, 1962) tem como base a equação eiconal, que é uma aproximação de alta freqüência (ótica geométrica), sendo inadequada quando o meio é altamente heterogêneo, apresentando problemas em modelos contendo interfaces com variação abrupta de curvatura (Trorey, 1970; Hilterman, 1970). Entretanto, com base no teorema de Green, gera resultados mais abrangentes, sendo útil para a modelagem de reflexões específicas (eventos). Métodos espectrais apresentam limitações para modelos com geometria complexa que apresentam fortes gradientes de propriedades físicas, como acontece em contatos de litologias distintas.
O uso do método de diferenças finitas (MDF) (Alterman e Karal, 1968; Ottaviane, 1971) permite o desenvolvimento de técnicas, com base na equação da onda, nas quais a geometria dos modelos não são aproximadas e nem limitadas. Desta forma, o método leva em conta não apenas as ondas diretas e refletidas (primárias e múltiplas), mas, também, as ondas: superficiais, refratadas, convertidas, difratadas, refletidas criticamente, e ondas observadas nas zonas de sombra da teoria do raio. O MDF permite, ainda, a obtenção de fotografias ("snapshots") do campo de ondas, em qualquer instante da propagação, sem qualquer custo adicional.

O MDF usa uma discretização do meio e, consequentemente, discretiza o campo de onda (amplitudes) que o atravessa, usando uma malha de pontos nos quais são lidos valores que representam parâmetros do meio a serem usados por uma equação recursiva discretizada da onda. Esse método usa aproximações das derivadas segundas espaciais e temporais obtidas por uma expansão de Taylor truncada, usando pontos vizinhos em diferenças ponderadas. Seus resultados, tão precisos quanto desejado, apresentam acurácia muito superior em comparação aos outros métodos de modelagem sísmica.

\section{Equação acústica da onda}

Para os objetivos deste trabalho, é suficiente considerar modelos que representem aspectos acústicos de meios geológicos. Pois, características distintivas observadas no uso de diferentes parametrizações na representação de modelos simples, tendem a acentuarem-se quando o grau de complicação do modelo aumenta.

A equação da onda de pressão é dada por

$\nabla^{2} p-\frac{1}{c^{2}} \frac{\partial^{2} p}{\partial t^{2}}=\vec{\nabla} \ln (\rho) \vec{\nabla} p$,

onde $p=p(\vec{x}, t)$ representa a pressão, $\vec{x}$ é o vetor posição, $t$ é o tempo, $c$ é a velocidade de propagação da onda de pressão em $(\vec{x}, t), \rho$ é densidade em $(\vec{x}, t)$, $\nabla^{2}$ é o laplaciano relativo às dimensões espaciais, $\vec{\nabla}$ é o operador gradiente e ln o logaritmo neperiano. Se $\rho$ é constante a equação (1) se reduz a

$\nabla^{2} p-\frac{1}{c^{2}} \frac{\partial^{2} p}{\partial t^{2}}=0$,

que é chamada de equação escalar acústica da onda. 


\section{Método de diferenças}

\section{Fundamentos}

Para substituir uma equação diferencial por uma outra equação na qual suas derivadas não são calculadas em seus limites infinitesimais e envolve somente diferenças e quocientes finitos, que é o cerne do método, faz-se necessário obter expressões que permitam calcular numericamente as derivadas. Isto pode ser feito via a série de Taylor, que para uma função $u=u(x)$, podemos escrever:

$$
u(x+\Delta x)=u(x)+\Delta x \cdot u^{(1)}(x)+\frac{(\Delta x)^{2}}{2 !} u^{(2)}(x)+\frac{(\Delta x)^{3}}{3 !} u^{(3)}(x)+\ldots,
$$

$u(x-\Delta x)=u(x)-\Delta x \cdot u^{(1)}(x)+\frac{(\Delta x)^{2}}{2 !} u^{(2)}(x)-\frac{(\Delta x)^{3}}{3 !} u^{(3)}(x)+\ldots$

Podemos obter a derivada segunda somando-se (4) com (3) e explicitando $u^{(2)}(x)$, assim:

$u^{(2)}(x)=\frac{u_{i+1}-2 u_{i}+u_{i-1}}{h^{2}}$.

Usando as equações (3) e (4) obtemos a seguinte expressão para a derivada segunda:

$\left(u_{x x}\right)_{i}=\frac{1}{12 h^{2}}\left[-u_{i-2}+16 u_{i-1}-30 u_{i}++16 u_{i+1}-u_{i+2}\right]$,

onde $u_{i+2}=u(x+2 \Delta x), \quad u_{i-2}=u(x-2 \Delta x)$ e $\left(u_{x x}\right)_{i} \quad$ é a derivada parcial segunda da função $u$ com respeito a $x$, calculada no nó $i$ da malha.

Usando a equação (5), obtemos a seguinte aproximação para as derivadas segundas temporais:

$\left(u_{t t}\right)_{k}=\frac{1}{h^{2}}\left[u_{k-1}-2 u_{k}+u_{k+1}\right]$,

Onde $\left(u_{t t}\right)_{k}$ é a derivada segunda da função $u$ relativa à variável $t$, calculada em $t=k \Delta t$, onde $\Delta t$ é o intervalo temporal.

\section{Procedimento Recursivo do MDF}

Utilizando-se as aproximações para as derivadas segundas representadas pelas equações (6) e (7), obtemos a seguinte expressão recursiva necessária à implementação computacional do MDF aplicado a equação da onda:

$u_{i, j}^{k+1}=\frac{1}{12}\left(\frac{c . \Delta t}{h}\right)^{2}\left[-\left(u_{i-2, j}^{k}+u_{i, j-2}^{k}\right)+16\left(u_{i-1, j}^{k}+u_{i, j-1}^{k}\right)+\right.$

$\left.-60 u_{i, j}^{k}+16\left(u_{i+1, j}^{k}+u_{i, j+1}^{k}\right)-\left(u_{i+2, j}^{k}+u_{i, j+2}^{k}\right)\right]+2 u_{i, j}^{k}-u_{i, j}^{k-1}+g$,

onde os índices $i, j$ e $k$ representam, respectivamente, as variáveis $x_{1}, x_{3}$ e $t$; e $g=-(c \cdot \Delta t)^{2} f$.

\section{Definição de modelos geológicos 2-D}

Procuramos focalizar nossos estudos em modelos sísmicos com grau de complexibilidade crescente. A Figura 1 apresenta o modelo $M_{I}$ (de três camadas com uma falha normal) e a Figura 2 apresenta o modelo $M_{I I}$ (de três camadas e uma bacia).

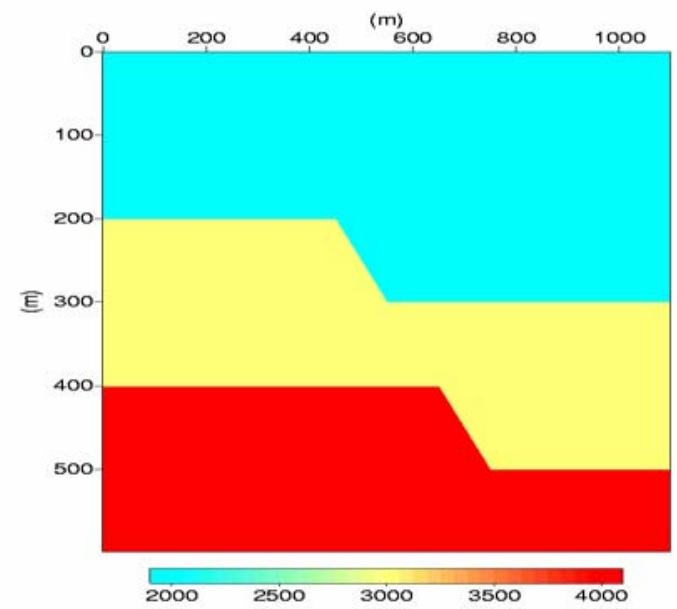

Figura 1. Modelo de campo velocidades sísmicas compressionais $\left(M_{I}\right)$ constituído por três camadas desalinhadas por uma falha geológica normal esquemática. As camadas são homogêneas e isotrópicas

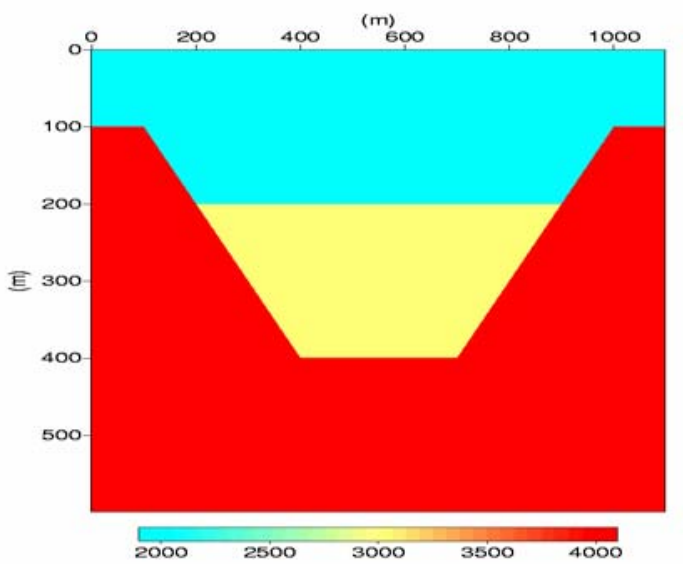

Figura 2. Modelo de campo de velocidades sísmicas $\left(M_{I I}\right)$ representando uma visão esquemática de uma bacia geológica. As diferentes regiões do modelo são homogêneas e isotrópicas.

\section{Parametrizações de campos de Velocidades sísmicas Divisão do Meio em Células}

A utilização de células (ou blocos) na representação do modelo é um tipo de parametrização bastante utilizado na modelagem sísmica, que é, simplesmente, a divisão do modelo geológico em blocos (células), onde cada uma possui uma velocidade constante. As Figuras 3 , e 4 
exibem, respectivamente, os modelos $M_{I}$, e $M_{I I}$; parametrizados por blocos (PB).

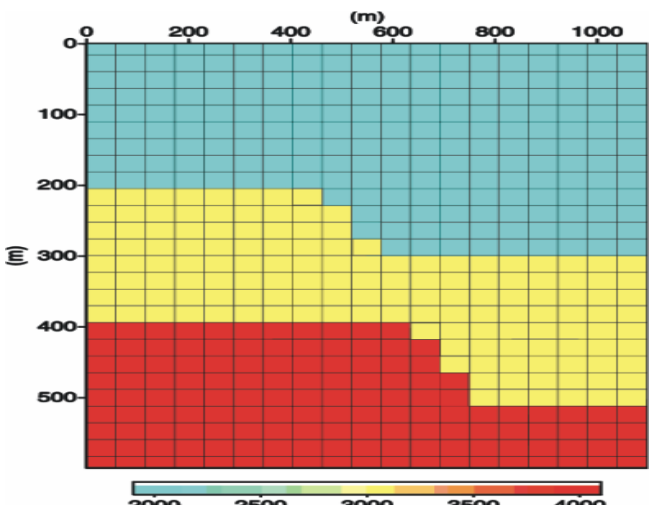

Figura 3. Modelo $M_{I}$ representado pela PB.

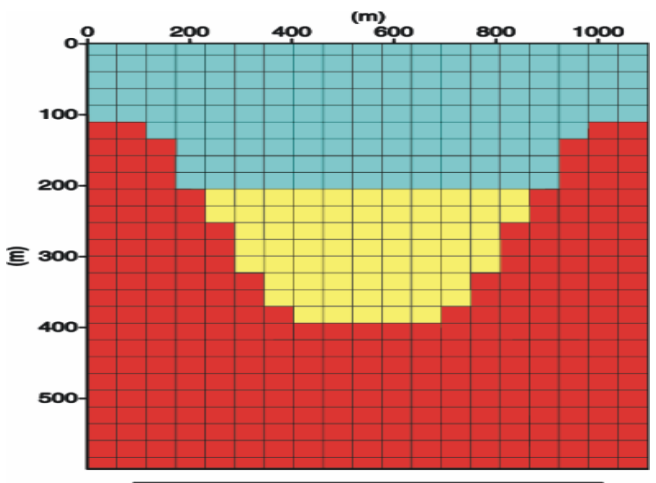

$2000 \quad 2500 \quad 3000 \quad 3500 \quad 4000$

Figura 4. Modelo $M_{I I}$ representado pela PB.

\section{Polinômio Trigonométrico}

A parametrização polinomial trigonométrica (PPT) consiste em representar o modelo geofísico através de uma única função polinomial trigonométrica. Tal representação é obtida através da particularização do teorema que fornece séries baseadas em funções ortogonais para funções de duas variáveis (Kreider et al., 1972). Isto é, o campo de velocidade sísmica $V(x, z)$ é representado por:

$V(x, z)=\sum_{i+j=0}^{N} \alpha_{i, j} f_{i}(x) g_{j}(z)$

onde

$f_{i}(x)=\frac{1}{2}\left\{\left[(-1)^{i}+1\right] \cos \left(\frac{i X}{2}\right)+\left[(-1)^{i+1}+1\right] \operatorname{sen}\left[\frac{(i+1) X}{2}\right]\right\}$, $g_{j}(z)=\frac{1}{2}\left\{\left[(-1)^{j}+1\right] \cos \left(\frac{j Z}{2}\right)+\left[(-1)^{j+1}+1\right] \operatorname{sen}\left[\frac{(j+1) Z}{2}\right]\right\},(1$

$X=\frac{\pi(2 x-l)}{l}$,

$Z=\frac{\pi(2 z-d)}{d}$,

$\alpha_{i, j}=\frac{\iint_{R} V(x, z) f_{i}(x) g_{j}(z) d R}{\iint_{R} f_{i}^{2}(x) g_{j}^{2}(z) d R}$,

onde $R$ é a região espacial na qual o modelo é considerado e $l$ e $d$ representam, respectivamente, o comprimento e as profundidades máximas do modelo. Tal função representa o campo de velocidade sísmica do modelo no qual a onda se propaga.

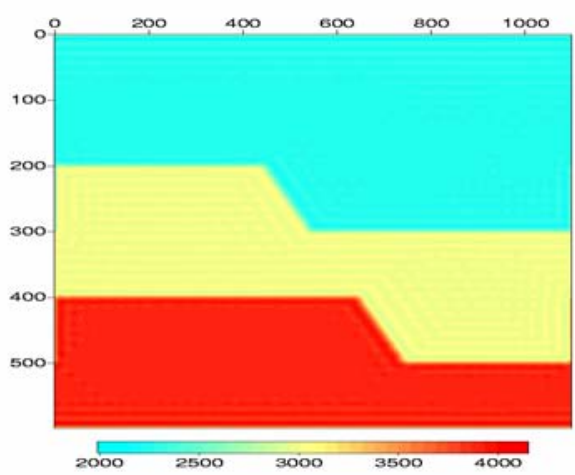

Figura 5. Modelo $M_{I}$ representado pela PPT.

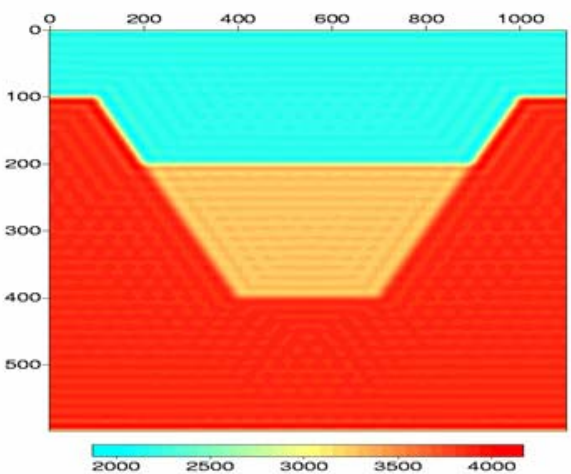

Figura 5. Modelo $\left(M_{I I}\right)$ representado pela PPT.

\section{Modelagem acústica 2-D}

Foram realizadas modelagens sísmicas (Dos Santos, 2002) baseadas em operadores escalares gerando quatro sismogramas para os dois modelos utilizando-se duas diferentes parametrizações consideradas para cada um deles. 
Para os modelos $M_{I}$ e $M_{I I}$, as Figuras 7 , e 8 mostram em (a) os sismogramas sintético obtidos por modelagem acústica aplicando o MDF à propagação da onda, usando blocos (PB), enquanto em (b), mostram aqueles produzidos quando os modelos são representados pela função polinomial trigonométrica.
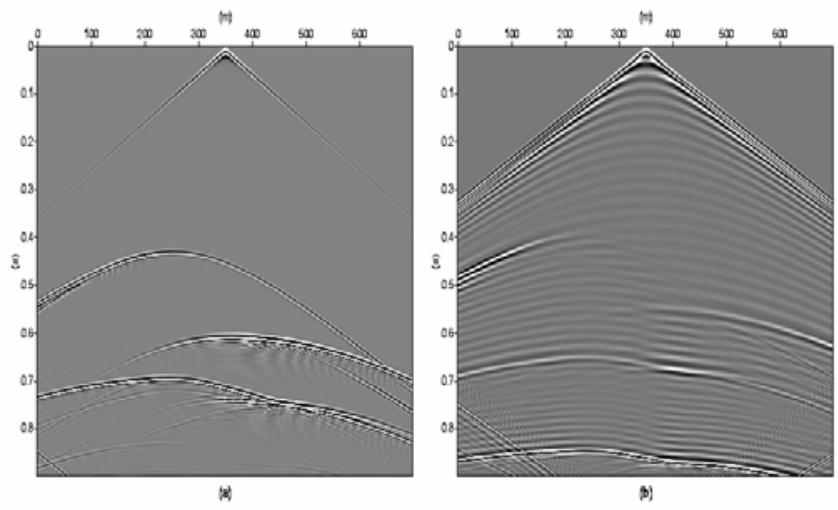

Figura 7. Sismogramas sintéticos referentes ao modelo $M_{I}$, usando: (a) PB, e (b) PPT. Em (a), a assimetria do modelo se manifesta nos sismogramas.
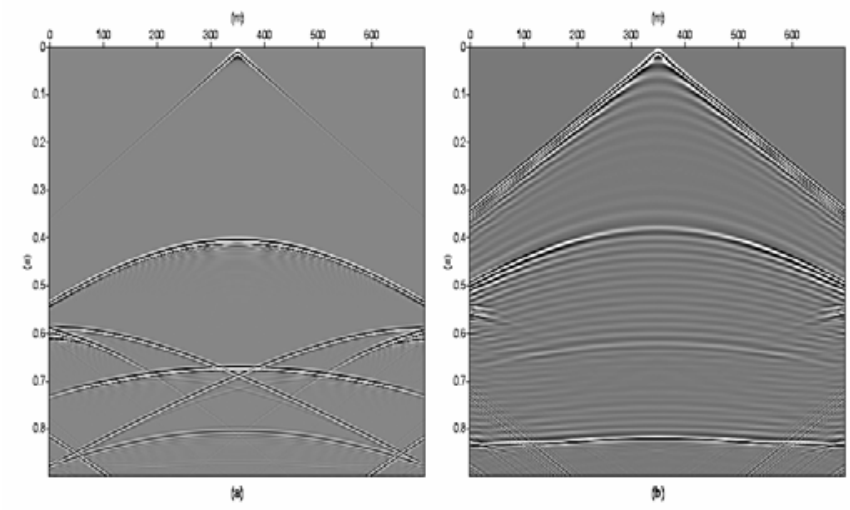

Figura 7. Sismogramas sintéticos referentes ao modelo $M_{I I}$, usando: (a) PB, e (b) PPT. Em (a), mais uma vez, vemos que no sismograma gerado usando PPT do modelo, muitos eventos são enfraquecidos pela grande quantidade de reflexões artificiais.

\section{Discussão e conclusões}

Em termos gerais, o algoritmo implementado por DF produziu bons resultados tanto para a PB como para a PPT.

Tanto na PB como na PPT, os sismogramas obtidos apresentam similaridades gerais nos resultados, salvo reflexões artificiais nos sismogramas gerados com a PPT que foram capazes de esconder muitos eventos que se manifestaram de forma clara quando a PB foi usada. Tais reflexões artificiais se devem ao fato do campo de velocidade representado por PPT oscilar mais.

A PPT proporciona uma maior economia de espaço de memória do computador, devido ao fato de não necessitar ter como entrada no programa de DF um arquivo de velocidade, pois estas no modelo podem ser calculadas durante a execução do programa, bastando entrar com a função polinomial trigonométrica que é definida por um número relativamente pequeno de coeficientes.

\section{Agradecimentos}

Os autores agradecem ao CPGG (Centro de Pesquisa em Geofísica e Geologia da Universidade Federal da Bahia), à ANP (Agência Nacional do Petróleo) e à CAPES; pelo apoio logístico e financeiro

\section{Referências}

Alterman, Z., \& Karal, F.C., 1968. Propagation of elastic wave in layered media by finite difference methods, Bull. Seis. Soc, v. 58, p. 367-398.

Červený, V., Molotov, I., e Pšenčík, I., 1977. Ray Method in Seismology. Praha, Univ. Karlova.

Dos Santos, R.H.M., 2002. Modelagem Acústica Bidimensional Usando Diferentes Parametrizações do Campo de Velocidades. Diss. de Mestrado, Geofísica, Uiversidade Federal da Bahia, Salvador, Brasil.

Hilterman, F.J., 1970. Three-dimensional seismic modeling. Geophysics, v. 35, p. 1020-1037.

Keller, J.B., 1962. Geometrical theory of diffraction. J. Opt. Soc. Am., v. 52, p. 116-130.

Kreider, D., Kuller, R.C., Ostberg, D.R., e Perkins, F. W., 1972. Introdução à Análise Linear. Editora Universal de Brasília, Rio de Janeiro.

Ottaviane, M., 1971. Elastic wave propagation in two evenly welded quarter spaces. Bull Seis. Soc. Am., v. 61, p. 1119-1152.

Trorey, A.W., 1970. A simple theory for seismic diffractions. Geophysisc, v. 47, p. 762-784. 